\title{
GOLPES Y BRAZOS EN CRUZ: EL CASTIGO ESCOLAR EN LA ESCUELA PÚBLICA FRANQUISTA (1938-1951)
}

\author{
Miriam Sonlleva Velasco ${ }^{1}$
}

\section{RESUMEN}

La disciplina escolar es uno de los fenómenos más presentes en la historia de la educación. La investigación que presentamos trata de conocer cómo fue vivida esta disciplina en las escuelas públicas franquistas. Para ello utilizamos el método biográfico-narrativo, asentado en cuatro relatos de vida de dos hombres y dos mujeres, que vivieron su escolaridad en España entre 1938 y 1951. Sus testimonios revelan cómo el maestro se presentó como la figura clave para el adoctrinamiento de los menores y sus actuaciones fueron respaldadas por las familias, la Iglesia y el Estado. Los castigos tanto físicos como psicológicos que sufrieron los escolares condicionaron su vida adulta y permanecen intactos en su memoria, dando muestras de cómo la educación autoritaria influye negativamente en las biografías de todas aquellas personas que la sufrieron durante su infancia.

Palabras clave: disciplina escolar, franquismo, infancia, memoria, oralidad.

${ }^{1}$ Universidad de Valladolid (UVa), Valladolid, Espanha. 


\section{GREVES E ARMAS NA CRUZ: O CASTIGO ESCOLAR NA ESCOLA PÚBLICA FRANQUISTA (1938-1951)}

\section{RESUMO}

A disciplina escolar é um dos fenômenos mais presentes na história da educação. A investigação que apresentamos tenta saber como esta disciplina foi vivida nas escolas franquistas públicas. Para isso, utilizamos o método biográfico-narrativo, baseado em quatro histórias de vida de dois homens e duas mulheres, que viveram sua escolaridade na Espanha entre 1938 e 1951. Seus depoimentos revelam como a professora foi apresentada como figura-chave da doutrinação de menores e suas ações foram apoiadas por famílias, pela Igreja e pelo Estado. As punições físicas e psicológicas sofridas pelos escolares condicionaram a vida adulta e permaneceram intactas em sua memória, mostrando como a educação autoritária influencia negativamente as biografias de todos os que sofreram durante a infância.

Palavras-chave: disciplina escolar, franquismo, infância, memória, oralidade.

\section{STRIKES AND ARMS IN CROSS: THE SCHOOL PUNISHMENT IN FRANCOIST PUBLIC SCHOOL (1938-1951)}

\section{ABSTRACT}

School discipline is one of the most present phenomena in the history of education. The investigation that we present tries to know how this discipline was lived in the public Francoist schools. For this, we use the biographical-narrative method, based on four life stories of two men and two women, who lived their schooling in Spain between 1938 and 1951. Their testimonies reveal how the teacher was presented as the key figure for the indoctrination of minors and their actions were supported by families, the Church and the State. The physical and psychological punishments suffered by schoolchildren conditioned their adult life and remain intact in their memory, showing how authoritarian education negatively influences the biographies of all those who suffered in the childhood.

Keywords: school discipline, francoism, childhood, memory, orality.

\section{COUPS ET ARMES EN CROIX : LES PEINES DE L'ECOLE PUBLIQUE FRANÇOIS (1938-1951)}

\section{RÉSUMÉ}

La discipline scolaire est l'un des phénomènes les plus présents dans l'histoire de l'éducation. L'enquête que nous présentons tente de savoir comment cette discipline a été vécue dans les écoles franquistes publiques. Pour cela, nous utilisons la méthode biographique et narrative, basée sur quatre histoires de vie de deux hommes et deux femmes, qui ont vécu leur scolarité en Espagne entre 1938 et 1951. Leurs témoignages révèlent comment l'enseignant était présenté comme la clé de l'endoctrinement des mineurs et leurs actions étaient soutenus par les familles, l'Église et l'État. Les punitions physiques et psychologiques subies par les écoliers ont 
conditionné leur vie d'adulte et sont restées intactes dans leur mémoire, montrant comment l'éducation autoritaire influence négativement les biographies de tous ceux qui ont souffert pendant leur enfance.

Mots-clés : discipline scolaire, franquisme, enfance, mémoire, oralité. 


\section{INTRODUCCIÓN}

Históricamente el niño ha sido considerado como un ser débil, dúctil y maleable que necesitaba ser instruido para su productividad (GUTIÉRREZ; PERNIL, 2004). Los problemas relacionados con sus impulsos instintivos precisaban la intervención de una mano adulta que le sirviera de guía. La escuela se ha presentado en la historia como la agencia garante de la atención educativa de la infancia y la responsable de su adoctrinamiento.

La institución educativa ha mantenido a lo largo de los años una serie de prácticas conservadoras con el fin de mantener la disciplina (WEAVER, 2012). La violencia no solo ha formado parte del quehacer pedagógico tradicional, sino que se ha considerado un instrumento didáctico indispensable para mantener el orden.

En la sociedad europea, a lo largo de la Edad Moderna, la violencia en la escuela era una práctica común. Foucault (1975) detalla que a partir del siglo XVIII se empezarán a imponer en la escuela un conjunto de técnicas, recogidas dentro de los reglamentos escolares, que permitirán un control minucioso del cuerpo. El autor denomina a este tipo de técnicas "disciplinas" que utilizan como instrumentos básicos la vigilancia jerárquica, el examen y el castigo disciplinario. Será a partir de entonces cuando el castigo en las escuelas comience a considerarse no solo como algo normal, sino incluso aconsejable (BIANCHINI, 2017). El fin de este tipo de sanciones no era otro que erradicar el error, la malicia, la inmoralidad y el vicio al que estaba abocado la infancia (HERRERA, 2013) y ejercer el poder, ante aquellos individuos que se salen de la norma, a través de la fuerza.

$\mathrm{Si}$ bien este tipo de prácticas fueron cambiando en la Edad Contemporánea y regulándose, algunos símbolos comunes se han mantenido con el tiempo. Por ejemplo, trabajos como los de Meda \& Brunelli (2018) o Weaver (2012) revelan como algunas prácticas como el uso de sombreros puntiagudos o aquellos caracterizados por atributos de animales como los 
burros, tan arraigados en la cultura popular de algunos países como Italia o Estados Unidos, han servido a lo largo de los años para humillar públicamente a todos aquellos escolares con algún problema de aprendizaje y para sancionar a los menores que se salieran de la norma.

Pero, además de este tipo de violencia psíquica, también se constata cómo en este periodo, en la escuela han estado presentes con frecuencia otras formas de violencia física. Bofetadas, manotazos, tirones de pelo y de orejas, golpes en las manos con una vara o con una regla de madera y empujones se han unido con otras penas corporales que provocan dolor - como ponerse durante un largo tiempo de rodillas, colocar los brazos en cruz y soportar el peso de algunos libros en las manos o estar atado a una silla -, para atemperar las inquietas conciencias infantiles y corregir sus malos hábitos (ROUSMANIERE; DEHLI; CONINCK-SMITH, 1997; SUÁREZ, 2004).

Autores como Verger (2008) ya destacan que el fenómeno de la violencia escolar, omnipresente en la historia de la educación, precisa de unos análisis minuciosos por la gran diversidad de formas que abarca. Además, se hace necesario contextualizar este fenómeno en función de la región en la que nace esa violencia y el momento histórico en el que se localiza.

En España, la obra de Luis Torrecilla revela cómo a lo largo de los siglos, los castigos tanto psíquicos como corporales fueron moneda corriente en los centros educativos. La costumbre de golpear y humillar a aquellos menores que incumplieran la regla fue socialmente aceptada hasta finales del siglo XIX (TORRECILLA, 1998).

A partir de 1876 comienzan a difundirse los ideales educativos de la Institución Libre de Enseñanza. El "alma" de la I. L. E., Francisco Giner de los Ríos, buscará la educación de la persona desde la delicadeza, el compromiso y la prudencia dando a la palabra el poder de corregir la indisciplina (RUIZ, 1993).

Giner y los hombres que le acompañan vuelven su mirada hacia la nueva pedagogía europea, especialmente hacia Rousseau, Pestalozzi y 
Froebel. [...]. Y no olvidan la idea de transformar España mediante una renovación moral que solo podía venir de la educación, dando lugar así a un proyecto educativo complejo, que mira desde luego hacia el interior del hombre, pero también hacia un nuevo modelo de sociedad (PUELLES, 2008, p. 46).

De este modo, en el periodo que se sitúa entre 1876 y 1936 se apostará por el destierro de los castigos en las escuelas y se comenzará a sembrar la semilla de una educación que abogue por la tolerancia, la educación integral y la coeducación (TORRECILLA, 1998, p. 117).

Pero el comienzo de la guerra civil dará fin a esta modernización de la educación española, caracterizada por ideas de armonía, integralidad y desarrollo de la plenitud. La década de los 40 vendrá acompañada por el desmantelamiento de la obra republicana, que según el Nuevo Gobierno "había envenenado" al pueblo español con doctrinas corruptas y le había llevado a la indisciplina (SÁNCHEZ-REDONDO, 2004).

El aparato escolar republicano fue así desplazado hasta su total eliminación y sustituido por una "nueva escuela" que reivindicaba los valores religiosos y patrios propuestos por la Iglesia y la Falange. De esta forma, la educación fue considerada como un vehículo privilegiado para transmitir la nueva ideología y garantizar el afianzamiento del régimen franquista (GONZÁLEZ, 2005, p. 414).

Para ello, se puso especial interés en formar a un modelo de maestro acorde con el binomio patria-religión que presidiera el modelo escolar del momento. Para tal fin, su preparación pedagógica y psicológica no solo pretendía dotarle de una cultura general armónica con los nuevos valores dictatoriales, para que posteriormente pudiera trasladarlos al ámbito educativo y al social; sino también convertirle en ejemplo de moralidad, vocación y autoridad (MARTÍN, 2015).

Pasados ochenta años desde el comienzo de la posguerra, aún permanece intacto en la memoria de los ancianos que vivieron su infancia en los 
primeros años de la dictadura franquista, el recuerdo de su experiencia escolar. Muchos guardan con recelo aquella vivencia sentida entre los temidos muros de la escuela. En sus testimonios descansa el recuerdo de una pedagogía autoritaria, cuyos efectos, tanto físicos como psicológicos, perduraron en el tiempo y aún permanecen vivos. Las cicatrices mentales que dejó aquel episodio violento, no solo se han instalado de forma permanente en la memoria de aquellas personas que lo padecieron, sino que, sin duda, han determinado su personalidad. El miedo, la humillación y la vergüenza siguen estando hoy presentes entre los rincones de sus recuerdos (VELÁZQUEZ, 2005).

Son varios los investigadores e investigadoras que dedican algún apartado de sus estudios a tratar el tema de la disciplina escolar en el franquismo (GRANA; ALONSO, 2009; GUICHOT, 2009, PÁRRAGA, 2010; SONLLEVA; TORREGO, 2014), pero son escasos los trabajos que analizan de forma precisa cómo fue impuesta en este periodo la disciplina en las aulas (SUÁREZ, 2004). Lo que sí se hace patente en todas estas investigaciones es la dureza de los castigos escolares impuestos y la naturalidad con la que los menores tenían interiorizada este tipo de violencia.

Knowles y Holt-Reynolds (1991) o Mollo (1984) hablan sobre la necesidad de rescatar relatos de escolares de otras épocas históricas como forma de ampliar aquellos acontecimientos que quedan oscurecidos por la escasez o la falta total de fuentes, como es el caso de la disciplina y el castigo escolar, así como sus repercusiones. En este artículo tratamos de avanzar en el conocimiento de esta línea de investigación y tomamos el testimonio de dos hombres y dos mujeres que vivieron su socialización primaria en plena posguerra (1938-1951), para conocer qué recuerdos tienen del castigo escolar y cómo aparece este fenómeno forjado en su memoria. A través de sus discursos intentaremos adentrarnos en el conocimiento de la figura del maestro y del régimen disciplinario que este imponía en el aula; analizaremos los tipos de castigos escolares descubiertos; y conoceremos la respuesta de padres y educadores ante el castigo escolar. 
Antes de comenzar a exponer los resultados descubiertos, tratamos de hacer una breve contextualización histórica sobre la educación en el franquismo y la concepción de la disciplina escolar en este momento histórico.

\section{DISCIPLINA Y CASTIGO ESCOLAR EN EL FRANQUISMO}

Es un tema conocido que la primera etapa de la dictadura franquista (1936-1951) aspiró a hacer del Estado el eje de toda la vida nacional. Inspirado en los modelos fascistas alemán e italiano, el Gobierno que nació tras la guerra civil tomó la educación como el estandarte de su política. Su finalidad fue la creación de un "hombre nuevo" y para ello se moldeó una pedagogía que otorgó a la religión un papel determinante. A ella se unieron otros valores, como la autoridad, la jerarquía, el orden o la disciplina (PUELLES, 2008, p. 106).

A pesar de que el nivel educativo primario no tuvo en sus comienzos un especial interés para el Nuevo Gobierno (más centrado en formar a la nueva élite que a las masas), desde el comienzo de la Guerra se empezó a divulgar en aquellas zonas donde triunfó la sublevación militar, como pensamiento pedagógico único, "la nueva escuela española". Fundamentada en pedagogos clásicos, en la doctrina eclesiástica y en los principios políticos de corte fascista, la nueva pedagogía negaba al niño la actividad, la espontaneidad y la libertad (POZO; BRASTER, 2012).

Con el debilitamiento de los fascismos aliados en el Eje el régimen franquista empezó a ser consciente del poder de la Educación Primaria para la transmisión de su ideología. Fue a partir de entonces, entrada la década de 1940, cuando la escuela se presentó como el medio idóneo por excelencia para el adoctrinamiento de las futuras generaciones y se empezó a planificar minuciosamente desde los libros de texto que debían ser utilizados en las aulas hasta las pautas de conducta, con el objetivo de que la infancia quedara 
impregnada de los valores patrios, tradicionales y cristianos (PALACIO; RUIZ, 1993, p. 23).

La Ley de Enseñanza Primaria de 17 de julio de 1945 sirvió para homogeneizar las pautas educativas de este nivel a nivel nacional. Con un marcado carácter católico e inspirado en la Divini Illius Magistri, el texto apuntaba que la instrucción primaria tenía como principal misión formar la voluntad, la conciencia y el carácter del niño en orden al cumplimiento del deber y a su destino.

La disciplina se presentará a lo largo de todo el texto legislativo como el principio rector de la pedagogía escolar, cuyo objetivo era conseguir un espíritu nacional fuerte y unido e instalar en el alma de las futuras generaciones la alegría y el orgullo de la Patria, de acuerdo con las normas del Movimiento y sus Organismos.

En este modelo educativo se consideraba al alumno como como un ser pasivo al que había que conducir en cada momento y adiestrar (PARRA, 2009), con el fin de que adquiriera ciertos hábitos sociales y respetara los principios dogmáticos del régimen. Para conseguir tal fin se ponía empeño en un modelo de educación forjado desde tres ejes: social (imprescindible para la formación del ciudadano); físico (necesario para el desarrollo fisiológico del escolar y como instrumento para su formación intelectual y moral); y profesional (como iniciación del alumno en su futura vida adulta y enfocado, para las niñas en la vida del hogar, la artesanía y la industria doméstica; y para los niños en la formación superior o el trabajo en la industria, el comercio o la agricultura).

Para el cumplimiento de este modelo educativo se decidió separar a los escolares según su sexo, aludiendo para ello a razones de orden moral y de eficacia pedagógica. La enseñanza primaria quedó a partir de entonces ordenada en un plan cíclico, que abarcaba desde los seis hasta los catorce años, y que se componía de tres tipos de contenidos: instrumentales (Lectura, Expresión gráfica y Cálculo), formativos (Religión, Geografía, Historia, Lengua Nacional, Matemáticas y Educación Física) y complementarios (Ciencias de la 
Naturaleza, Música, Canto, Dibujo, Trabajos manuales, Prácticas de taller y labores femeninas).

Sin menospreciar las formas autoritarias de enseñanza de todos aquellos contenidos, una de las asignaturas a las que el Estado prestará una atención especial para la formación de un cuerpo dócil es la Educación Física. En el artículo diez de la citada ley puede leerse como a esta materia no solo le atañe el cultivo de las prácticas higiénicas, sino la formación de una juventud fuerte, sana y disciplinada. A partir de esta materia, el cuerpo será entendido como carente de voluntad individual y se pondrá al servicio de lo colectivo. Así, el hombre, sano y vigoroso, será formado para su contribución en la construcción física del nuevo orden; mientras el cuerpo de la mujer deberá ser educado para cumplir con la función que le ha sido encomendada: la reproducción y el cuidado de las nuevas generaciones (COTERÓN, 2012).

En este ambiente de instrucción disciplinar, el estudiante se convirtió en el eje a formar, instruir y adiestrar, a quien le correspondió ser dominado y alienado mediante la orden, la imposición, la represión, la cohibición, el castigo y la repetición. La desobediencia fue manejada, atajada y hasta clausurada con todo tipo de amenazas, intimidaciones y castigos (LOAIZA, 2012, p. 24).

El control absoluto sobre las conductas, el carácter y los comportamientos de los niños y jóvenes buscaban no solo el perfeccionamiento estético del individuo, sino también el de la raza hispana, una de las grandes obsesiones del régimen dictatorial (MAURI, 2016). Por ello, no se dudará en prescribir en el texto legislativo base de la Educación Primaria la forma de entender la disciplina infantil:

La disciplina en las escuelas primarias será eminentemente activa, se amoldará a la edad escolar y tendrá carácter preventivo. El maestro, en la corrección de los niños buscará la colaboración de los padres y demás educadores. En ningún caso podrán emplearse castigos que de palabra o de obra supongan ludibrio o humillación afrentosa (LEY PRIMARIA DE 1945, Art. 100). 
De este modo, el maestro, considerado como una autoridad suprema con tintes divinos - gracias a su posición y al reconocimiento que el régimen le otorgó -, tenía el control absoluto sobre los estudiantes y podía ejercer sobre ellos la violencia que considerara oportuna en aras de corregir cualquier mal hábito o conducta indisciplinada.

Por supuesto, la labor de la familia consistía en apoyar activamente la labor docente en la formación del carácter y la personalidad del niño, por ello, se hacía necesario que la institución familiar continuara con las medidas disciplinarias impuestas por el maestro, que tenían como último fin corregir sus defectos, encaminar sus hábitos y estimular en el menor el gobierno de sí mismo.

En este contexto disciplinario el menor se veía sometido desde la infancia a acatar la norma. De esta suerte, la disciplina se constituyó en una tecnología de poder cotidiana que penetró en los cuerpos infantiles como un arma de control (MAURI, 2016).

\section{METODOLOGÍA}

La investigación que presentamos trata de responder a la siguiente pregunta de investigación: ¿cómo fue vivido el castigo escolar por los estudiantes de escuelas públicas nacionales en la posguerra española (19381951)?

Para poder responder a dicho interrogante tomaremos el método biográfico-narrativo como metodología de investigación. Nuestro interés es adentrarnos en la propia vivencia del castigo escolar desde los modos de sentir de aquellos niños y niñas que lo padecieron de forma directa. Para ello, nos serviremos de cuatro relatos de vida realizados a dos hombres y dos mujeres españoles, cuya experiencia escolar se contextualiza entre 1938 y 1951 en escuelas públicas nacionales. 
Entendemos por relato de vida una forma particular de entrevista, la entrevista narrativa, en la que un investigador pide a una persona, en un momento determinado de su historia, que le cuenta su experiencia biográfica (BERTAUX, 2005). Esta forma de hacer conocimiento científico apuesta por dar voz a los sujetos, aproximándose a determinadas vivencias sociales desde la individualidad (ARIAS; ALVARADO, 2015). A pesar de esta individualidad, es innegable que dichas narrativas personales rescatan una experiencia contextualizada en un periodo determinado, dentro de una nación concreta y de un grupo social explícito, por tanto, forman parte de la historiografía y de la colectividad en la que nacen.

Encarar una investigación desde la metodología biográfico-narrativa implica aproximarse a los postulados epistemológicos de la hermenéutica; y supone una apuesta ética y política particular para el investigador, dado su papel activo en la construcción de conocimiento (ARIAS; ALVARADO, 2015). Además, este tipo de investigación no busca la representatividad ni la generalización de resultados (BASSI, 2014); sino la posibilidad de apertura de nuevas construcciones teóricas a partir de un juicio crítico sobre los resultados (BOTERO; ALVARADO; LUNA, 2009).

Estos presupuestos de partida obligan a la persona que realiza el estudio a explicitar cuáles han sido los pasos que se han seguido en la construcción de conocimiento, como trataremos de hacer a continuación.

Nuestra investigación se contextualiza en la provincia de Segovia (España). Según datos de Instituto Nacional de Estadística, en el curso académico 1947/1948, el número de estudiantes matriculados en escuelas nacionales de Primera Enseñanza en España era de 2.765.047 alumnos; y el de escuelas privadas ascendía a 640.934 escolares. En la provincia de Segovia los datos son para la misma periodicidad de 34.018 escolares matriculados en escuelas públicas y 2.170 alumnos matriculados en centros privados (menos de un 6\% del total). Estas cifras nos sirvieron para seleccionar a los informantes de acuerdo a las siguientes características: hombres y mujeres nacidos en la década 
de 1930 en la provincia de Segovia; cuya experiencia escolar estuviera contextualizada en escuelas nacionales de Primera Enseñanza (según la tipología de centros citada en la Ley 17 de julio de 1945 sobre Educación Primaria); y nacidos en familias de clase popular².

Para el primer contacto con las protagonistas del estudio se recurrió a redes de contacto familiares. Después de las oportunas explicaciones en relación a la investigación, se realizó un consentimiento sobre los datos del estudio, en el que primaba la protección de los testimonios de los participantes y la fidelidad de sus versiones.

En el siguiente Cuadro 1 mostramos un resumen biográfico de cada participante:

Cuadro 1 - Datos biográficos de las participantes.

\begin{tabular}{|l|l|l|l|}
\hline PARTICIPANTE & \multicolumn{1}{|c|}{$\begin{array}{c}\text { PRESENTACIÓN } \\
\text { BIOGRÁFICA }\end{array}$} & $\begin{array}{l}\text { SITUACIÓN } \\
\text { TIPO Y AÑOS DE } \\
\text { ESCOLARIZACIÓN }\end{array}$ & $\begin{array}{l}\text { PERSONAL } \\
\text { CUANDO } \\
\text { REALIZÓ EL } \\
\text { RELATO }\end{array}$ \\
\hline Felisa & $\begin{array}{l}\text { Mujer, nacida en 1937 en } \\
\text { Hontoria (Segovia). Después } \\
\text { de la escuela aprende labores. } \\
\text { Contrae matrimonio a los 22 } \\
\text { años. Tiene tres hijas. En su } \\
\text { etapa adulta se dedica a } \\
\text { ayudar a su marido en las } \\
\text { tareas del campo y es ama de } \\
\text { casa. }\end{array}$ & $\begin{array}{l}\text { Escolarizada ocho años } \\
\text { (1943-1951) en una } \\
\text { escuela nacional rural. } \\
\text { Su aula - } \\
\text { exclusivamente } \\
\text { femenina- está } \\
\text { tutelada por una } \\
\text { maestra. }\end{array}$ & $\begin{array}{l}\text { Reside en } \\
\text { Jubilad. Viuda. } \\
\text { Segovia. }\end{array}$ \\
\hline Gregorio & $\begin{array}{l}\text { Hombre, nacido en 1933 en } \\
\text { Segovia. Tras la escuela } \\
\text { trabaja en una fábrica. } \\
\text { Contrae matrimonio a los 28 } \\
\text { años. No tiene hijos. Ejerce }\end{array}$ & $\begin{array}{l}\text { Escolarizado cuatro } \\
\text { años (1941-1946), en } \\
\text { un colegio nacional } \\
\text { masculino. Convive } \\
\text { toda su escolaridad con }\end{array}$ & $\begin{array}{l}\text { 80 años. } \\
\text { Casado. } \\
\text { Jubilado. } \\
\text { Reside en } \\
\text { Segovia. }\end{array}$ \\
\hline
\end{tabular}

2 Entendemos por clase popular en el periodo franquista al grupo formado por familias de trabajadores urbanos y rurales sometidos a problemas de empleo, altos niveles de mortalidad, vivienda precaria y problemas de acceso a la educación (MAZA, 2002). 


\begin{tabular}{|c|c|c|c|}
\hline PARTICIPANTE & $\begin{array}{l}\text { PRESENTACIÓN } \\
\text { BIOGRÁFICA }\end{array}$ & $\begin{array}{l}\text { TIPO Y AÑOS DE } \\
\text { ESCOLARIZACIÓN }\end{array}$ & $\begin{array}{c}\text { SITUACIÓN } \\
\text { PERSONAL } \\
\text { CUANDO } \\
\text { REALIZÓ EL } \\
\text { RELATO }\end{array}$ \\
\hline & $\begin{array}{l}\text { diversas profesiones entre las } \\
\text { que destaca la de camionero. }\end{array}$ & maestros varones. & \\
\hline Paula & $\begin{array}{l}\text { Mujer. Nacida en } 1931 \text { en } \\
\text { Segovia. A la edad de nueve } \\
\text { años empieza a trabajar } \\
\text { cuidando niños y limpiando } \\
\text { viviendas. Contrae } \\
\text { matrimonio a los } 24 \text { años. } \\
\text { Tiene } 3 \text { hijos y } 2 \text { hijas. } \\
\text { Continúa trabajando como } \\
\text { personal de limpieza en la } \\
\text { etapa adulta. }\end{array}$ & $\begin{array}{l}\text { Escolarizada tres años } \\
\text { (1938-1941) en una } \\
\text { escuela graduada mixta } \\
\text { de la capital, dentro de } \\
\text { un aula femenina. } \\
\text { Convive toda la } \\
\text { escolaridad con } \\
\text { maestras. }\end{array}$ & $\begin{array}{l}86 \text { años.Viuda. } \\
\text { Jubilada.Reside } \\
\text { en Segovia. }\end{array}$ \\
\hline Antonio & $\begin{array}{l}\text { Hombre. Nacido en 1933, en } \\
\text { un pueblo alejado de la capital } \\
\text { segoviana. A los } 14 \text { años } \\
\text { comienza su labor en la } \\
\text { agricultura, en la que } \\
\text { permanecerá hasta su } \\
\text { jubilación. Contrae } \\
\text { matrimonio a los } 27 \text { años. } \\
\text { Tiene un hijo y una hija. }\end{array}$ & $\begin{array}{l}\text { Escolarizado ocho años } \\
\text { (1939-1947) en una } \\
\text { escuela nacional rural. } \\
\text { Su aula - } \\
\text { exclusivamente } \\
\text { masculina- está } \\
\text { tutelada por un } \\
\text { maestro. }\end{array}$ & $\begin{array}{l}84 \text { años.Viudo. } \\
\text { Jubilado. } \\
\text { Reside en } \\
\text { Segovia. }\end{array}$ \\
\hline
\end{tabular}

Fuente: elaboración propia.

La recogida de información se realizó en las viviendas de los protagonistas entre los años 2013 y 2017. Las 21 entrevistas que componen los cuatro relatos de vida fueron transcritas y devueltas a cada narrador para que pudiera eliminar aquella información que no considerase oportuna para su publicación. La técnica para la recogida de datos se basó en la entrevista semiestructurada. Para recomponer cada relato realizamos una batería de 75 preguntas, distribuidas en cuatro bloques temáticos correspondientes con: la infancia, la escuela, el trabajo y la vida adulta. La información recogida fue analizada de acuerdo con un enfoque hermenéutico, privilegiando en un primer momento la singularidad de cada historia relatada; para después realizar un 
análisis global de los datos y trazar correspondencias (CORNEJO; MENDOZA; ROJAS, 2008).

Esta primera categorización de datos, nos ayudó a reducir el volumen de los mismos y a aproximarnos a nuestro objeto de estudio (PATTON, 2002). De esta forma, nacieron tres unidades de significado, que se corresponden con: el maestro y su forma de entender la disciplina escolar; la tipología de castigos subyacente; y la respuesta de padres y otros educadores ante el castigo escolar. A partir de estas unidades relacionamos otros temas vinculados, que se verán expuestos en el apartado de análisis.

Los datos orales sido sometidos a un proceso de validación a través de legislación educativa (1936-1951), que aparecerá comentada en el apartado de resultados como un dato más del análisis. Para la redacción del informe final irán apareciendo extractos de las entrevistas, en diálogo con las interpretaciones de los autores y los resultados de otros investigadores, formando una trama narrativa (ATKINSON; COFFEY, 2003).

\section{ANÁLISIS DE DATOS}

\section{“LE TENÍAMOS UN POCO DE PÁNICO”: EL MAESTRO COMO AGENTE DE AUTORIDAD}

Desde nuestras primeras entrevistas y el recuerdo que los protagonistas tienen de la figura del maestro, advertimos cómo el docente se convirtió para la infancia de clase popular en un referente educativo de primer orden. Parece ser que la cultura de la que hacía gala este agente, junto con el poder social que el franquismo le otorgó resultaban ser dos aspectos clave para tener un reconocido valor social entre la población. 
El maestro era una persona representativa y se le respetaba mucho, tanto por parte de la familia como de los niños [...]. Se les veía más formados, mejor valorados en la sociedad (Felisa).

La Ley Primaria de 1945 especifica en su artículo cincuenta y siete que el maestro "debe estimar su vocación como servicio a Dios y a la Patria y merecer y exigir para su profesión respeto y consideración pública”. No es casualidad que todos nuestros participantes hablen del respeto que no solo ellos, sino la propia ciudadanía, tenían hacia esta figura docente. De acuerdo con el orden social establecido, el maestro era considerado como un padre dentro de la institución educativa y a él se le debía la educación de los pequeños (HERRERA, 2013).

El maestro era un hombre serio y honrado. Yo le tenía más respeto que a mi padre (Antonio).

Las primeras descripciones que los participantes hacen de los maestros que los acompañaron durante la escolaridad, siempre vienen precedidas, cuando les nombran, por los vocablos “don" y “doña”. Estas expresiones de respeto y distinción social constituyen un aspecto destacable en sus testimonios, pues solo son verbalizadas cuando los protagonistas hablan del maestro o del sacerdote, y nos dan las primeras pautas para conocer quiénes eran los educadores principales de la infancia.

Uno se llamaba Don Desiderio, que tenía el pie derecho más cortito que el izquierdo [...] y me acuerdo que tenía una barba muy, muy larga. Ese maestro era muy mayor. Luego estuve con otro que se llamaba Don Alfredo, que procedía de Valseca, y otro que se llamaba Don Lucio (Gregorio).

El extracto de entrevista de Gregorio, va mostrando algunas de las características físicas y morales del maestro que se hacen sentir en otros testimonios. Además, el docente solía caracterizarse por una forma de vestir 
tradicional y acorde con los patrones de género impuestos por el régimen, y acompañaba sus actuaciones del buen ejemplo y la prudencia (LOZAIZA, 2012).

La maestra era una mujer muy buena [...]. Nos esperaba cada mañana dentro de clase (Paula).

El sexo del maestro será un factor clave para determinar sus cualidades personales. De este modo, mientras la maestra es el fiel reflejo de la religiosidad y de la moralidad; al maestro se le atribuye la responsabilidad, el orden y la transmisión del patriotismo:

De política no, este señor no quería meterse para nada en las cosas de la política, no se hablaba nada en clase. Él era mucho de Franco y mucho de la Falange (Antonio).

Desde el relato de la relación entre docentes y estudiantes comenzamos a ver cómo el maestro se convierte en agente de autoridad en el aula. Su misión no solo era educar a los estudiantes a nivel cultural, sino también moral. La disciplina se convertirá en una herramienta privilegiada para la formación del carácter (VIGARELLO, 2005).

Te pegaban y te castigaban, había que respetar a maestros, curas y autoridad, aunque había chicos muy traviesos (Felisa).

$\mathrm{Su}$ autoridad y poder en el aula se hacen visibles en todos los testimonios. La arbitrariedad de sus conductas deja constancia de la impronta imborrable de sus actuaciones.

Iba así paseando por los pasillos de la clase, viendo quién hacía los deberes o no los hacía, te ponía la mano encima de la cabeza, como 
que te iba a acariciar, le mirabas y a lo mejor le preguntabas - ¿qué pasa? - (pensando que te iba a acariciar). Te cogía y te pegaba un tirón de orejas. Le decías - profesor, ¿̇por qué me ha hecho eso?, si yo no he hecho nada ahora- y te decía - ipero lo he hecho yo!, y como soy el que mando aquí, hago lo que me da la gana- (Gregorio).

La entrevista de nuestro participante nos lleva a ver que la autoridad del docente y su legitimidad no solo se dejaban ver a través de sus características y cualidades personales, sino también de la impunidad de la que gozaba. Penetrando en este recuerdo, vamos descubriendo cómo en el aula no se establecía un clima afectivo; ni tampoco era frecuente establecer ningún diálogo personal entre docente y estudiantes.

La maestra no era muy cariñosa. Nos dábamos los buenos días por la mañana y nos despedíamos por la tarde, pero no había contacto físico... No había abrazos. Esta maestra no era mala, pero vamos, si te tenía que meter un tortazo, te le metía (Felisa).

Los menores tenían asumido cuál era el lugar que ocupaban dentro del aula y cómo se encontraban sometidos a la autoridad del maestro. Por ello, es frecuente escuchar a los participantes hablar de cómo respetaban la disciplina impuesta, siendo este respeto garantía de buena educación.

Yo en la escuela nunca me he portado mal. Las niñas éramos muy buenas y muy tranquilas. Nos pasábamos el tiempo de clase bien sentaditas, escuchando lo que la maestra nos decía y escribiendo en el cuaderno que llevábamos lo que nos mandaban y ya está. Sabíamos que al colegio se iba a aprender y a callar y ninguna niña se movía de su sitio (Paula).

En todos los narratorios vemos cómo la jornada escolar se organizaba atendiendo a una serie de rutinas. Los escolares entraban al aula santiguándose y dando los buenos días al maestro. Después se colocaban en su correspondiente puesto escolar, que había sido asignado por el docente al principio del curso en 
función de las capacidades del menor.

Pues le decíamos - ibuenos días Don... Fulano! -. Nos iba apuntando y nos metíamos a clase a sentarnos, porque cada uno teníamos asignado ya nuestro sitio, así que ya sabíamos dónde teníamos que ir, y de ese sitio no nos movían en todo el curso (Gregorio).

A continuación, era frecuente rezar algunas oraciones y trabajar de forma individual las asignaturas instrumentales hasta la hora del recreo.

$\mathrm{Al}$ entrar en clase, nos santiguábamos, rezábamos algún rezo y luego nos sentábamos y nos poníamos a trabajar hasta la hora del recreo. Luego salíamos a ese pequeño patio que te contaba a jugar un ratillo y así hasta la hora de comer (Paula).

En aquellas escuelas nacionales en las que estaban escolarizados niños y niñas, en este tiempo de recreo se seguía manteniendo un modelo segregador. Siguiendo el ejemplo de sus propios maestros, los escolares se relacionaban con otros menores del mismo sexo.

Las niñas solo tenían contacto con la profesora que estaba a cargo de su clase o con las otras maestras de las otras clases. La verdad es que, aunque estábamos en un colegio al que iban chicas y chicos, nosotras no teníamos ningún contacto ni con los niños ni con sus profesores (...). Desde pequeños nos enseñaban que estar juntos, niños y niñas en la escuela, era un pecado, y ya sabes que los maestros tenían que dar ejemplo (Paula).

Después del recreo, se trabajaban otras asignaturas formativas como la Historia o la Geografía. En el horario vespertino, para las niñas se ofrecía un amplio repertorio de conocimientos centrados en labores femeninas y para los niños, se primaba la adquisición de conocimientos complementarios de carácter artístico (Música y Dibujo) o utilitario (Trabajos Manuales). Terminada la jornada escolar, los menores recogían su puesto y rezaban de nuevo algunas 
oraciones. Las salidas de la escuela se hacían formando una fila y, uno a uno, cada escolar iba despidiéndose de su maestro.

Cuando se terminaba la clase, la maestra nos mandaba recoger y nos decía -adiós niñas, hasta mañana-. Nosotras, una por una, la íbamos diciendo "adiós señorita, adiós señorita, adiós señorita..." todas. Cuando se llegaba la hora de irnos a casa, hacíamos una fila todas las niñas, ordenadas allí en el pasillito, y cuando llegábamos a la puerta de salida, cada una se iba a su casa (Paula).

Curiosamente, la labor del maestro - principalmente en zonas rurales se deja ver también fuera del horario escolar. La legislación sobre Educación Primaria decretada en 1945, da muestras de cómo el docente tenía entre sus deberes acompañar a los escolares, a los oficios religiosos, tarea que hemos comprobado en los testimonios recogidos; pero, además, el docente vigilaba las conductas de sus estudiantes durante el tiempo libre.

Un día se enfadó muchísimo. Tenía yo catorce años y había en el pueblo la costumbre de que teníamos que pagar en el baile si queríamos bailar con alguna chica. Nosotros estábamos siempre en la puerta, viendo cómo bailaban los demás, porque éramos pequeños; pero, un día, les preguntamos a los chicos más mayores si podíamos entrar... Entonces ellos nos dijeron que sí, pero que teníamos que pagar una botella de vino. Pues, claro, nosotros lo hicimos y al día siguiente el señor maestro nos echó una regañina que no veas... Y nos dijo - ianalizad esta frase! "el alcoholismo perturba la salud y la moral de los seres humanos" ianalizadla! - iA nosotros nos tenía firmes, no te creas! (Antonio).

Serán este tipo de conductas autoritarias y disciplinarias, tanto dentro como fuera del espacio escolar, las que lleven a los participantes a sentir algunas sensaciones de angustia y desconfianza hacia la figura docente.

Pues sí [...], yo era un crío y le tenía respeto, le teníamos un poco de pánico (Gregorio). 


\section{“LA LETRA CON SANGRE ENTRA": HACIA UNA TIPOLOGÍA DE LOS CASTIGOS}

La legislación primaria franquista de 1945 dedica su artículo cien a la disciplina infantil, apuntando a que ésta debía ser activa, amoldada a la edad y que tendría un carácter preventivo. Desde las primeras entrevistas con los participantes vemos cómo los castigos físicos y psicológicos solían ser una constante en el aula. Los propios protagonistas, cuando verbalizan la vivencia del castigo, naturalizan este tipo de sanciones.

Anda mujer... si no te entraba era normal, ¿no? Es de la única manera que te entran las cosas, dice el refrán que "la letra con sangre entra" y así es... (Felisa).

A partir de los datos recogidos hemos podido diferenciar dos tipos de castigos en el aula. Por un lado, aparecen aquellos que surgen del incumplimiento de las obligaciones académicas, ya bien sea por la negación del estudiante hacia la tarea o por problemas de aprendizaje. Y por otro lado, afloran aquellos castigos que están orientados a modificar cualquier conducta que vulnere las normas disciplinarias. En relación a la primera tipología de castigos, los académicos, vemos cómo los pequeños muestran al principio de la escolaridad algunos problemas con el cálculo matemático y las reglas básicas de alfabetización. La lectura se presenta en las primeras edades, como una de las actividades con mayores dificultades para los estudiantes y un foco de continuas amenazas a su integridad física.

A leer aprendimos con una cartilla [...] sentadas al lado de la señorita, que nos metía unos cartillazos en la cabeza si no te entraban las letras... Eso sí, te daba con la cartilla en la cabeza... Tampoco te podía hacer mucho daño, hombre, pero... sí, sí te daba (Felisa).

Junto a la lectura, la escritura parece ser otra de las destrezas que 
presenta mayores problemas en el aula. Cuando los protagonistas narran sus experiencias en el aprendizaje de la escritura observamos ciertas conductas que llaman nuestra atención, por su carácter antipedagógico:

En aquellos años eso de que alguien fuera zurdo en el colegio no se permitía, a nosotros nos obligaban a aprender con la derecha para escribir y para todo (Paula).

Profundizando en esta forma de enseñar, vemos cómo frecuentemente se castigaba a los menores que utilizaban la mano izquierda, atándoles la mano a la espalda o a una bolsa para que no lo hicieran. Este tipo de actitudes apuntan una falta de formación específica del profesorado sobre el conocimiento de la infancia, con las consiguientes repercusiones que esta ausencia produce en el menor.

Lo que hacían para enseñarles con la derecha era atarles la mano izquierda a una bolsa, le ataban la mano y así ya no podía escribir con esa mano... Entonces, al niño que era zurdo, como te digo, le ataban un talego a la muñeca, como se decía, una bolsita... y entonces ya no podía escribir, el que era diestro pues seguía igual. Además, es que era obligatorio aprender con la mano derecha a hacer las cosas y a escribir. Además, a los niños que escribían con la izquierda se les regañaba mucho, si eras zurdo te regañaban pero bien (Felisa).

Cuando los escolares eran instruidos en la lectura y la escritura, los maestros comenzaban a profundizar en el aprendizaje del cálculo y las reglas ortográficas, contenidos con especial dificultad para los escolares, criados en el seno de familias en muchos casos sin alfabetizar. La equivocación en la escritura y las primeras operaciones matemáticas conllevaba para los estudiantes el inmediato castigo por parte del docente. La reiterada copia de la palabra o la cuenta errónea en la pizarra - bajo la burla de los compañeros -, la negación de su disfrute lúdico en el horario de recreo y el reiterado golpeo en los dedos con una regla de madera, suelen ser las sanciones más utilizadas para que los 
pequeños aprendan de sus errores.

Cuando nos preguntaba una cosa y no sabíamos contestarle, nos mandaba salir a la pizarra. Salíamos a la pizarra y, a lo mejor, nos mandaba escribirlo varias veces. Me acuerdo que cuando trabajábamos en Lengua las reglas ortográficas, por ejemplo, cómo se escribe buey o vaca, nos mandaba copiarlo catorce o veinte veces en la pizarra, para que no se nos olvidara [...]. Y si no lo hacías bien... pues ya sabes, a la regla la mano y palo va, palo viene (Gregorio).

Otra de las asignaturas en las que se da muestras de un gran autoritarismo es la Educación Física, con la que se pretendía formar una juventud fuerte, sana y disciplinada. Para ello, se formó a un grupo de personas especialmente vinculadas con el proyecto falangista y se programaron actividades físicas con claro interés político (MANRIQUE, 2014). En las zonas rurales esta materia no aparece en los recuerdos de los escolares, pero su presencia sí se hace notar en el testimonio de la escuela nacional urbana masculina. La evocación de la figura del maestro de Educación Física nos dibuja a una persona con un carácter extremadamente autoritario, que solía imponer su fuerza y poder en el aula:

Lo que sí que hacíamos era gimnasia [...] con un profesor que tenía una uva el tío...Me acuerdo que tenía un florete, con el que practicaba esgrima, y al que le veía un poco más, así, o que no le gustaba lo que estaba haciendo, iplas! Un latigazo con el florete, en las costillas o donde le pillara, le daba igual [...]. Allí en el gimnasio, pues nos dedicábamos a mover un poco el cuerpo. Estirábamos los brazos hacia delante, las piernas las movíamos, nos agachábamos, nos levantábamos y cuatro cosas... que lo hacíamos bien, pues bien, que lo hacíamos mal, pues ya estaba el profesor para darnos y que lo hiciéramos bien (Gregorio).

La narración de los protagonistas también nos lleva a considerar los espacios escolares. Será a través de los mismos donde podamos ver cómo el aula dispone de lugares destinados al castigo. Las esquinas de clase se convertirán en 
zonas donde los menores serán separados del grupo; pero, además, el lugar que cada escolar ocupe dentro del aula será identificado con su rendimiento académico. Los narradores comentan cómo los niños y niñas más aventajados ocupaban los puestos de las primeras filas; mientras aquellos "más torpes" se sentaban en la parte de atrás, siendo objeto de mofa entre sus compañeros.

Junto a estos castigos académicos aparecen otros morales, que nos llevan a ver cómo no solo era necesario ser un alumno ejemplar a nivel académico, sino que también era importante no salirse de las normas establecidas. En relación a este aspecto, los testimonios desvelan cómo eran especialmente castigadas las conductas irrespetuosas hacia el maestro.

\begin{abstract}
Nos pegaba con la regla varias veces y si se la escondías, pues iay Goyo para que quieres más! Y eso que yo estaba en la última fila, Decía iGoyo!, iCallejo!, salga usted aquí. ¿Dónde está mi regla? -; y yo le decía - no la he visto - Cogía y decía - ¿no la has visto? Pues muy bien... hala, ponte hay contra la pared, los brazos en cruz - y te los ponía llenos de libros. Nos amenazaba y nos decía - iComo tires los libros te machaco! - . Se me cansaban los brazos y los dejaba caer y, bueno, majo, me ponía en una esquina, donde más sólo estuviera, para que no me distrajera con los demás. Y, a mí, palos con la regla me ha dado que no te imaginas, todos los que ha querido (Gregorio).
\end{abstract}

Sentados de rodillas con los brazos en cruz llenos de libros; tirones de pelo y de oreja; golpes con la regla en los dedos; e incluso, ataques con algunos objetos del aula, como nos cuenta una de las protagonistas, solían ser algunas de las sanciones habituales ante tal falta de respeto.

\footnotetext{
Los chicos se reían del maestro algunas veces y luego los castigaba y, bueno, me acuerdo que a los chicos el maestro les mandaba poner las manos cerradas con los dedos hacia arriba y les daba con la regla. Pero, claro, no me extraña, le ponían chinchetas en la silla y le hacían de rabiar un montón... y, claro, luego que les daba con el timbre en la cabeza... normal (Felisa).
}

Pero los menores no solo eran sancionados cuando faltaban al respeto al 
maestro, sino también cuando perturbaban el silencio del aula, molestaban a sus compañeros, no controlaban su voz, no se sentaban correctamente en la silla.

\begin{abstract}
Me acuerdo que cuando nos pegábamos o nos insultábamos, nos separaba la señorita y... bueno, de ahí no solía pasar, aunque a veces si habíamos hecho alguna cosa mala, nos ponía con los brazos en cruz, de rodillas, un buen rato... imenudo cansancio! Ese era nuestro castigo, $\mathrm{y}$, a veces, no era por pelearnos, sino por estar hablando entre nosotras o por haber hecho alguna cosa mal o haber regañado con una compañera (Felisa).
\end{abstract}

Otra de las conductas que eran seriamente reprendidas era cualquier comunicación que se pudiera establecer dentro del entorno escolar entre niños y niñas. Las participantes explican que no era frecuente establecer ningún tipo de contacto entre sexos dentro del centro y que, si algún escolar se aproximaba al grupo de niños del sexo contrario, el maestro le castigaba sin salir al patio.

Los niños y las niñas, aunque a veces jugábamos a la misma hora en el patio, no nos relacionábamos, ni siquiera nos saludábamos. Eso era un pecado, hija (Paula).

Ante cualquier tipo de conductas contrarias a la norma y su correspondiente castigo, el menor debía mostrar un severo arrepentimiento. La falta de este remordimiento suponía sufrir nuevas represalias.

Una vez sí que pegó a dos o tres chicos. No sé si estaría de mal humor, o qué, porque a uno que no lloraba, le debió de pegar bien con el palo de una silla (Antonio).

El menor debía acatar la sanción impuesta, pues el docente era la máxima autoridad en el aula (SUÁREZ, 2004) y estaba protegido tanto por la ley como por la institución a la que servía. 
Llegaba un momento que el peso se hacía insoportable y los brazos se te caían, quisieras o no... Y, como se te cayeran, te pegaba unos reglazos en la mano, para que te espabilaras... Y no te estoy hablando de tener los libros cinco minutos, una vez me tuvo veinte minutos...tenía unos dolores en los brazos que le dije - iyo no me pongo más tiempo así!, castígueme como usted quiera, pero no me castigue con los libros porque me duelen mucho los brazos - y me dijo - aquí mando yo -; y le dije - pues no te preocupes, que se lo voy a decir ahora mismo al Director - . Y me dijo - se lo puedes decir a quien quieras, pero hoy el que manda aquí soy yo, y te he preguntado una cosa del temario y no me la has sabido contestar, te he castigado con esto y ya verás como no se te va a olvidar, así que ihala!, sigue con los libros - (Gregorio).

Todos los testimonios recogen la resignación con la que los participantes vivían la autoridad del maestro y las medidas disciplinarias que este imponía en el aula.

iY que ibas a hacer, hija mía! Si rechistabas te iba a pegar más... Era mejor callarse y dejarle que hiciera lo que le diera la gana (Gregorio).

Este tipo de violencia sufrida llevó a uno de los participantes incluso a abandonar la escuela, ante la impotencia sentida por el trato recibido y el miedo a la autoridad.

Ya le digo, a mí me pegaban mucho y no quise seguir allí estudiando, me fui de la escuela elemental y me puse a trabajar (Gregorio).

\section{“LAS FAMILIAS NO SE METÍAN": PADRES Y EDUCADORES ANTE EL CASTIGO ESCOLAR}

Muchas familias de clase popular, durante la dictadura franquista, no podían ofrecer a la descendencia una educación de calidad. Algunos participantes explican que uno o sus dos progenitores eran analfabetos, por lo 
que era habitual que la alfabetización de los menores la dejaran a cargo del maestro.

Las familias dejaban la educación a cargo del maestro y en eso no se metían, lo único que nos enseñaban era a obedecer y a respetar (Felisa).

Será este encargo a la figura docente, mucho más culta y con mayor reconocimiento social, la que haga que las familias tengan un especial respeto hacia el mismo.

Los padres tenían respeto a los maestros y como la mitad de ellos, pues los hombres no sabían nada de letras, pues yo creo que hasta les parecía el maestro alguien superior a ellos (Felisa).

Parece ser que los contactos que se establecían entre las familias y el docente eran muy esporádicos a lo largo de la escolaridad.

La familia y la escuela eran dos ambientes separados. Los padres no iban a la escuela, no se metían en lo que hacíamos allí (Felisa).

Generalmente, la madre solía ser la encargada de establecer el primer contacto con el maestro, el día que tenía que formalizar la matrícula del menor en el centro, y este trato no se volvía a establecer hasta el final de la escolaridad.

Cuando nos iban a llevar al colegio pues sí, mi madre llevaba los papeles que hicieran falta y luego ya no volvió más por allí. Eso mi madre... mi padre, no, jamás [...]. Mi madre fue la responsable de matricularnos y acompañarnos el primer día, luego ya nos juntábamos algunas chicas del barrio y ya íbamos y veníamos solas (Paula).

En las zonas rurales alejadas a de la capital, los maestros y maestras 
solían establecer su lugar de residencia en el propio pueblo en el que ejercieran su labor profesional, como recomendaba la legislación primaria de 1945. Será esta experiencia la que les permita mantener el contacto con los vecinos de la localidad.

Jugaba los domingos a la partida con los hombres y se enfadaba si perdía... El maestro estaba muy integrado en el pueblo, era muy buen maestro (Antonio).

Los testimonios nos dejan ver que la labor que el docente realizaba dentro del aula no solía ser cuestionada por las familias.

Los padres no se enfadaban como ahora. No creas que iban a reclamarle nada al maestro, no. Si les regañaba por algo sería, ¿̇no? Y era la verdad... ahora un maestro no puede regañar ni decir nada a un niño, pero antes... le podía meter un cachete y los padres no decían nada... ha cambiado la vida en todos los sentidos (Felisa).

Precisamente, la legislación educativa del momento argumenta que la familia debía participar activamente con el maestro para la formación del carácter y la personalidad del niño. Además, debía estar de acuerdo con las medidas disciplinarias que el docente considerara pertinentes para corregir los defectos del menor, encaminar sus hábitos y estimular en él el gobierno de sí mismo.

Yo cuando llegaba a casa, a lo mejor, llegaba llorando o... doliéndome las manos, o lo que fuera y... mi madre me decía ¿qué te ha pasado? Pues... que me han pegado madre, y ella me decía, iclaro! Ya habrás hecho alguna... y la decía yo, pues como todos, madre, yo que sé, me han pegado reglazos en las manos y se me ha ocurrido que para que no me pegase más, la solución era esconderle la regla al maestro detrás del armario. Y mi madre me decía, claro, es que eso no se puede hacer, hijo, eso no se puede hacer (Gregorio). 
Las familias tomaban el castigo infantil como algo natural y necesario. Los mismos participantes explican que, habitualmente, los padres defendían cualquier actitud que el docente llevara a cabo dentro de la escuela y enseñaban a los pequeños a respetar las normas impuestas por el maestro.

Los padres, casi, en vez de defender a los hijos, defendían al maestro. Mis padres me decían "tú hijo, cállate y haz lo que te manden, haces las tareas y todo lo que ellos te manden", desde luego a aguantar y a callar, eso era lo que nos enseñaban nuestros padres (Gregorio).

La relación que el maestro tenía con otros poderes locales, principalmente la mantenida con el sacerdote, se hace notar en todos los testimonios - especialmente en aquellos de entornos rurales -.

La relación entre los maestros y el cura era muy buena. Se ponían de acuerdo para programar los contenidos que nos iban a enseñar. Me acuerdo que iba muchas veces por la escuela a hablar con los maestros (Felisa).

Los castigos que el docente imponía a los escolares, en relación a la vulneración de algunas normas morales, también eran apoyados por el cura. La legislación educativa de posguerra ya hace alusión a la necesaria complementariedad entre escuela e Iglesia para formar la voluntad, la conciencia y el carácter del niño.

Recuerdo que esto nos lo decía el sacerdote cuando íbamos a los ejercicios, que tuviéramos mucho cuidado de juntarnos con los chicos, que no jugáramos con ellos. Nosotras debíamos tener nuestros juegos, juegos de niñas y ellos los suyos, juegos de niños y estar juntos era un pecado... iY cuidado como te vieran juntos! Te regañaban un montón, tanto las maestras como el cura (Paula). 


\section{DISCUSIÓN Y CONCLUSIONES}

Según el análisis de datos realizado hemos observado que el maestro, durante la dictadura franquista, como ocurre en Italia o Alemania, países que vivieron bajo una política totalitaria en la segunda mitad del siglo $\mathrm{XX}$, era considerado una autoridad, tanto dentro como fuera del aula (LOPARCO, 2017). A través de métodos coercitivos el docente conseguía el respeto social que merecía su profesión.

Pero el maestro no solo era consciente de su posición y reconocimiento, sino también de la necesidad de acompañar sus órdenes con una buena imagen $\mathrm{Su}$ forma de vestir, su vida dentro y fuera de la institución, así como sus actuaciones y comportamientos sociales transmitían un perfil que estaba en consonancia con sus predicaciones (LOZAIZA, 2012, p. 25).

La disciplina en las escuelas era rigurosa y esta era posible gracias a una serie de rutinas que se realizaban a lo largo del horario escolar y permitían el adoctrinamiento de los menores en los valores patrióticos y religiosos impuestos por el régimen (NARGANES, 2011).

Las conclusiones extraídas en el estudio realizado por Schüerof y Gaspar (2017) en Brasil y contextualizado entre 1910 y 1940, se asemejan con las encontradas en nuestra investigación. Para garantizar la disciplina en el aula, el castigo no solo no era considerado un acto violento, sino que las actitudes represivas hacia la infancia eran razonadas como las mejores formas de inculcar buenas conductas. Se trataba de una violencia tolerada por la propia institución escolar, que otorgaba al maestro la responsabilidad de formar una juventud disciplinada y capaz de someterse a las órdenes del poder (VERGER, 2008).

Parece ser que la práctica del castigo escolar estaba generalizada en las escuelas españolas de posguerra - tanto públicas como privadas - y así lo muestran algunas investigaciones como las de Grana y Alonso (2009), Guichot (2009) o Peinado (2012). Autores como Velázquez (2005) advierten que la agresión que ejerce el adulto sobre el niño puede llegar a ser tan sutil que pasa 
desapercibida, llegando a formar parte de su vida cotidiana, como ha ocurrido con nuestros protagonistas. Los participantes han dado muestra de cómo naturalizaban e incluso apoyaban cualquier sanción contraria a la norma llevada a cabo dentro del aula.

Pero nuestro trabajo refleja cómo los efectos que implican este tipo de sanciones violentas perduran en el tiempo. La amplia gama de correctivos a los que fueron sometidos los participantes durante su trayectoria escolar, tanto por el incumplimiento de sus obligaciones académicas como por la vulneración de las normas disciplinarias del aula, los han llevado a tener un recuerdo amargo de su experiencia escolar (GRANA; ALONSO, 2009). Insultos, humillaciones verbales, golpes, separaciones del grupo de iguales y agresiones físicas y psicológicas aparecen hoy en su memoria y dan muestras de la influencia que este tipo de agresiones les generaron en su vida personal (VELÁZQUEZ, 2005).

Además, el análisis evidencia cómo la escuela, en contacto con otras instituciones educadoras como la Iglesia, se encargaron de que el periodo de socialización primario facilitara la asimilación de una serie de normas sexuales a través de la disciplina, los castigos y la creación del complejo de culpabilidad. Como afirman nuestras participantes, el contacto entre niños y niñas durante el horario escolar suponía "un pecado". Investigaciones como las de Badillo (1990) ya hablan de la enorme carga emocional que imprimió la educación femenina en este periodo como forma para que las alumnas asumieran la represión y reprodujesen el rol sumiso que debían desempeñar en la etapa adulta.

La respuesta de las familias ante el régimen disciplinario que vivían los menores en el aula pasaba por el refuerzo de la autoridad docente (LOZAIZA, 2012). Los padres de familias populares, con un nivel cultural inferior al del maestro y algunos de ellos víctimas del analfabetismo, confiaban en esta figura la educación de sus hijos y reconocían al docente como el único e irrefutable conocedor de los saberes.

La relación que el maestro mantenía con los poderes locales y el poder social que el régimen franquista le confió fueron elementos clave para que la 
población más humilde sintiera cierta reserva, timidez y temor a contradecir las guías disciplinarias que el docente imponía tanto dentro como fuera del aula. Las familias enseñaban a la descendencia a aceptar la disciplina impuesta por el maestro y a obedecer sus órdenes. Como explicaba uno de los participantes los padres solían decir "tú hijo, cállate y haz lo que te manden". Este tipo de actitudes de respaldo favorecían un clima escolar propenso a la violencia que influía en las experiencias infantiles (VELÁZQUEZ, 2005).

Concluimos este estudio haciéndonos eco de la importancia de investigar la influencia de instrumentos disciplinarios en la escuela en la trayectoria biográfica de escolares de épocas pasadas. Investigaciones como la de Aragão y Gonçalves (2012) dan muestras de cómo el castigo escolar genera un alto nivel de ansiedad entre los estudiantes que lo padecen y repercute de forma determinante en sus conductas en la vida adulta. Resulta innegable que un clima escolar propenso a la violencia excluye y limita a todos aquellos niños y niñas que lo sufren y deja una profunda huella en su memoria.

El trabajo realizado despeja futuras líneas de investigación que sería necesario abordar. El análisis del castigo escolar en el franquismo a través de manuales escolares o la recuperación de otras fuentes escritas e iconográficas para su tratamiento podría ser una de las guías metodológicas que avanzaran nuevos resultados. Asimismo, podría ser conveniente analizar en conjunto los castigos a los que fue sometido el colectivo infantil por parte de la familia, de la Iglesia y de la escuela, como forma de conocer la complementariedad disciplinaria que imponían dichas instituciones y descubrir las repercusiones de estas pautas educativas en la trayectoria biográfica de quienes las padecieron. El tema del castigo escolar se presenta en nuestros días como una línea de investigación prolífica en el campo de la historia de la educación.

\section{REFERENCIAS}


ARAGÃO, Milena; GONÇALVES, Anamaria. Práticas de castigos escolares: enlaces históricos entre normas e cotidiano. Conjectura: filosofia e educação, v. 17, n. 2, p. 17-36, 2012.

ARIAS, Ana María; ALVARADO, Sara Victoria. Investigación narrativa: apuesta metodológica para la construcción social de conocimientos científicos. CES Psicología, Medellín, v. 8, n. 2, p. 171-181, 2015.

ATKINSON, Paul; COFFEY, Amanda. Encontrar el sentido a los datos cualitativos. Estrategias complementarias de investigación. Medellín, Colombia: Editorial Universidad de Antioquia, 2003.

BADILLO, Rosa María. La construcción del pensamiento femenino en una sociedad agraria durante el franquismo: Istán (Málaga). Espacio, tiempo y forma - Serie V - Historia Contemporánea, n. 3, p. 69-84, 1990.

BASSI, Javier Ernesto. Hacer una historia de vida. Decisiones clave en el proceso de investigación. Athenea Digital, n. 14, v. 3. p. 129-170, 2014.

BERTAUX, Daniel. Los relatos de vida. Perspectiva etnosociológica. Barcelona: Bellaterra, 2005.

BIANCHINI, Paulo. Cattivi maestri: la violenza fisica e psicologica nella scuola moderna. In: DI POL REDI, Sante.; COGGI, Cristina. (Ed.). La Scuola e l'università tra passato e presente. Volume in onore del Prof. Giorgio Chiosso. Milano: FrancoAngeli, 2017. p. 37-52.

BOTERO, Patricia; ALVARADO, Sara; LUNA, María Teresa. La comprensión de los acontecimientos políticos ¿Cuestión de método? Un aporte a la investigación en las Ciencias Sociales. In: TONON, Graciela (Ed.). Reflexiones

latinoamericanas sobre investigación cualitativa. Buenos Aires: Prometeo, 2009. p. 148-201.

CORNEJO, Marcela; MENDOZA, Francisca; ROJAS, Rodrigo. La investigación con relatos de vida: pistas y opciones del diseño metodológico. Psykhe, v. 17, n. 1, p. 29-39, 2008.

COTERÓN, Javier. La educación física en los primeros años del franquismo (1939-1945). Materiales para la historia del deporte, n. 10, p. 1-11, 2012. FOUCAULT, Michel. Surveiller et punir. París: Éditions Gallimard, 1975.

GRANA, Isabel; ALONSO, Mariana. La educación de las mujeres en Andalucía durante el franquismo a través de las historias de vida. In: BERRUEZO, María Reyes; CONEJERO, Susana (Coord.). El largo camino hacia una 
educación inclusiva: La educación especial y social del siglo XIX a nuestros días. XV Coloquio de historia de la educación, Pamplona-Iruñea. PamplonaIruña: Universidad Pública de Navarra, 2009, p. 101-112.

GONZÁLEZ, Teresa. La educación insular durante el franquismo. Tebeto: anuario del Archivo Histórico Insular de Fuerteventura, n. 18, p. 411-436, 2005. GUICHOT, Virginia. La cultura escolar del franquismo a través de la historia oral. Cuestiones pedagógicas, n. 20, p. 215-245, 2009.

GUTIÉRREZ, Aurora; PERNIL, Paloma. Historia de la infancia. Itinerarios educativos. Madrid: Uned, 2004.

HERRERA, Claudia Ximena. Castigos corporales y escuela en la Colombai de los siglos XIX y XX. Revista iberoamericana de educación, n. 62, p. 69-87, 2013.

KNOWLES, Gary; HOLT-REYNOLDS, Diane. Shaping pedagogies through personal histories in preservice teacher education. Teachers college record, v. 93, n. 1, p. 87-113, 1991.

LOAIZA, Yasez Eder. La disciplina en las escuelas normales de Caldas en las décadas de 1960-1970: El poder y la autoridad para vigilar y castigar.

Magistro, v. 6, n. 12, p. 17-38, 2012.

LOPARCO, Fabiana. Former teachers' and pupils' autobiographical accounts of punishment in Italian rural primary schools during Fascism. History of education, v. 46, n. 5, p. 618-630, 2017.

MANRIQUE, Juan Carlos. Actividad física y juventud en el franquismo (19371961). Revista internacional de medicina y ciencias de la actividad física y el deporte, v. 14, n. 55, p. 427-449, 2014.

MARTÍN, Bienvenido. La cultura escolar y el oficio de maestro. Educación XXI, v. 18, n. 1, p. 147-166, 2015.

MAURI, Marta. Disciplinar el cuerpo para militarizar a la juventud. La actividad deportiva del frente de juventudes en el franquismo (1940-1960). Historia crítica, n. 61.

MAZA, Elena. La España de Franco. Madrid: Actas, 2002.

MEDA, Juri; BRUNELLI, Marta. The dumb child: contribution to the study of the icogenesis of the dunce cap. History of education \& children's

literature, v. 13, n. 1, p. 41-70, 2018. 
MOLLO, Stizane. Souvenirs d'école. In : POL, Enric et al. (Ed.). Vers un millor entorn escolar. Barcelona: Universitat de Barcelona, 1984 p. 197205 .

NARGANES, José Claudio. Infancia y lectura en la educación franquista. Clave XXI - Reflexiones y experiencias en Educación, n. 4, p. 1-37, 2011.

PALACIO, Irene; RUIZ, Cándido. Infancia, pobreza y educación en el primer franquismo. Valencia: Universitat de València, 1993.

PARRA, José María. La evolución de la enseñanza primaria y del trabajo escolar en nuestro pasado histórico reciente. Tendencias pedagógicas, Madrid, v. 14, p. 145-158, 2009.

PÁRRAGA, Carmen. Educación durante el franquismo. Temas para la Educación - Revista digital para profesionales de la enseñanza, n. 11, p. 1-16, nov. 2010.

PATTON, Michael. Qualitative research \& evaluation methods. California: Sage, 2002.

PEINADO, Matilde. Enseñando a señoritas y sirvientas. Formación femenina y clasismo en el franquismo. Madrid: Catarata, 2012.

POZO, María del Mar; BRASTER, Sjaak. El movimiento de la Escuela Nueva en la España franquista. Revista brasileira de história da educação, v. 12, n. 3, p. 15-44.

\section{PUELLES, Manuel de. Política y educación en la España}

contemporánea. Madrid: Uned, 2008.

ROUSMANIERE, Kate; DELHI, Kari; CONINCK-SMITH, Ning. Discipline, moral regulation and schooling: a social history. New York-London:

Routledge, 1997.

RUIZ, Julio. Francisco Giner de los Ríos (1839-1915). Perspectivas, v. 23, n. 34, p. 808-821, 1993.

SÁNCHEZ-REDONDO, Carlos. Leer en la escuela durante el franquismo. Cuenca: Ediciones de la Universidad de Castilla-la Mancha, 2004.

SCHÜEROF, Dilce; GASPAR, Vera Lucía. "Não obedeceu, pode punir”: castigos escolares no ensino público primário catarinense (1910-1940). Pedagógica, v. 19, n. 41, p. 194-208, 2017.

SONLLEVA, Miriam; TORREGO, Luis. La escuela primaria del primer 
franquismo desde las voces del alumnado segoviano: una iniciación en la investigación educativa. Tendencias pedagógicas, n. 24, p. 285-306.

SUÁREZ, Mercedes. Los castigos y otras estrategias disciplinarias vistos a través de los recuerdos escolares. Revista de educación, n. 35, p. 429-443, 2004.

TORRECILLA, Luis. Niñez y castigo. Historia del castigo escolar. Valladolid: Universidad de Valladolid, 1998.

VELÁZQUEZ, Luz María. Experiencias estudiantiles con la violencia en la escuela. Revista mexicana de investigación educativa, v. 10, n. 26, p. 739764, 2005 .

VERGER, Jacques. École et violence: faits, percepción, discours. Histoire de l'éducation, n. 118, v. 2, 2008.

VIGARELLO, George. Corregir el cuerpo. Historia de un poder pedagógico. Buenos Aires: Nueva Visión, 2005.

WEAVER, Heather A. Object lessons: a cultural genealogy of the Dunce cap and the apple as visual tropes of american education. Paedagogica historica, v. 48, n. 2, p. 215-241, 2012.

MIRIAM SONLLEVA VELASCO é Doctora en Educación. Licenciada en Pedagogía. Graduada en Magisterio de Educación Infantil y Primaria. Master en Investigación en Educación y Psicología Infantil. Actualmente contratada postdoctoral en el Departamento de Pedagogía de la Universidad de Valladolid.

E-mail: miriam.sonlleva@uva.es

(iD) http://orcid.org/0000-0001-6268-8670

Recebido em: 21 de outubro de 2018

Aprovado em: 01 de maio de 2019 
